JOURNAL of

MAINE MEDICAL CENTER Journal of Maine Medical Center

Volume 2

Issue 1 Volume 2, Issue 1 (January 2020)

Article 10

2020

\title{
Toxic leukoencephalopathy presenting as agitation and social withdrawal in a patient with substance use disorder: a case report
}

Andrew R. Beauchesne

Tufts University School of Medicine, Boston, Massachusetts; Maine Medical Center, Portland, Maine

Et al.

Follow this and additional works at: https://knowledgeconnection.mainehealth.org/jmmc

Part of the Neurology Commons

\section{Recommended Citation}

Beauchesne, Andrew R. and King, Brian F. (2020) "Toxic leukoencephalopathy presenting as agitation and social withdrawal in a patient with substance use disorder: a case report," Journal of Maine Medical Center. Vol. 2 : Iss. 1 , Article 10.

Available at: https://knowledgeconnection.mainehealth.org/jmmc/vol2/iss1/10 https://doi.org/10.46804/ 2641-2225.1035

The views and thoughts expressed in this manuscript belong solely to the author[s] and do not reflect the opinions of the Journal of Maine Medical Center or MaineHealth.

This Case Report is brought to you for free and open access by Maine Medical Center Department of Medical Education. It has been accepted for inclusion in the Journal of Maine Medical Center by an authorized editor of the MaineHealth Knowledge Connection. For more information, please contact Dina McKelvy mckeld1@mmc.org.

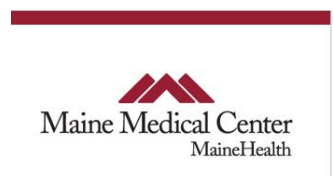


Toxic leukoencephalopathy presenting as agitation and social withdrawal in a patient with substance use disorder: a case report

Authors

Andrew R. Beauchesne and Brian F. King 


\title{
Toxic leukoencephalopathy presenting as agitation and social withdrawal in a patient with substance use disorder: a case report
}

\author{
Andrew R. Beauchesne ${ }^{1,2}$, Brian F. King, MD² \\ ${ }^{1}$ Tufts University School of Medicine, Boston, MA, ${ }^{2}$ Maine Medical Center, Portland, ME
}

\begin{abstract}
Introduction: $\quad$ Toxic leukoencephalopathy (TLE) is characterized by damage to myelin of cerebral white matter and is caused by several agents, including drugs of abuse. The clinical presentation of TLE is variable and not fully understood.
\end{abstract}
Clinical findings: A 51-year-old male presented with pneumonia, parapneumonic effusion, and acute kidney injury. His medical course was complicated by neurobehavioral changes, including aggression and social withdrawal.

\begin{abstract}
Diagnoses, Interventions, and Outcomes:

Acute toxic leukoencephalopathy was diagnosed based on diffuse subcortical T2-weighted-fluidattenuated inversion recovery (T2/FLAIR) on brain magnetic resonance imaging, bilateral frontal arrhythmic delta waves on encephalogram, and a history of polysubstance abuse.
\end{abstract}

Conclusions:

\begin{abstract}
This case suggests that TLE should be included in the differential diagnosis for patients with a history of substance abuse and neurobehavioral changes, including agitation and social withdrawal. It also emphasizes the need to identify and treat patients with substance use disorder to prevent complications.
\end{abstract}

Keywords:

toxic leukoencephalopathy, substance use disorder, neurology

A 51-year-old male welder with a history of polysubstance use self-presented with fever, dyspnea, lower-extremity edema, joint aches, "mud-brown urine", and oliguria. He was recently hospitalized at an outside hospital for community acquired pneumonia complicated by parapneumonic effusion and acute kidney injury. He left against medical advice to be closer to his home in Maine. The patient reported no significant past medical history but declared polysubstance use, including tobacco, alcohol, marijuana, cocaine, and oral street opiates. On admission to our hospital, his neurologic and psychiatric exam was normal. A urine toxicology screen was positive for tetrahydrocannabinol (THC), cocaine, and opiates.

Correspondence: Andrew Beauchesne

Tufts University School of Medicine

Boston, MA 02111

Andrew.Beauchesne@tufts.edu
His hospital course included chest tube insertion for a loculated pleural effusion and tunneled dialysis catheter for hemodialysis. On hospital day 18, he exhibited neurobehavioral changes, including paranoia (e.g., accusing nursing staff of "spying" on him and "micromanaging" his medication schedule) and aggression (e.g., yelling and throwing bloody gauze at health care providers). He withdrew from his care team and treatment plan by refusing most medications, blood draws, vitals, and meals; locking himself in the bathroom for hours multiple times per day; being unwilling or unable to answer questions; and refusing psychiatric evaluation. On hospital day 25 , the patient became incontinent of urine and developed ataxic gait and tremor of the left upper extremity. Further testing was performed to assess new-onset neuropsychiatric and neuromotor functions, including routine lab work; an analysis of ammonia, thyroid-stimulating 
hormone (TSH), vitamin B12, and folate levels; testing for treponema; urine toxicology; a lorazepam challenge test to exclude catatonia; and a head computed tomography (CT) scan. Findings from these assessments were unremarkable. Acute toxic leukoencephalopathy (TLE) was diagnosed based on diffuse subcortical T2-weighted-fluidattenuated inversion recovery (T2/FLAIR) signals on brain magnetic resonance imaging (MRI) (Figure 1), bilateral frontal arrhythmic delta waves on encephalogram, and a history of polysubstance abuse. His treatment plan included divalproex and continual reassessment of his ability to make decisions and care for himself. He failed to improve and eloped on hospital day 88.

\section{DISCUSSION}

TLE is characterized by damage to myelin of cerebral white matter. It is caused by several offending agents, including cranial irradiation, antimetabolites, immunosuppressants, antifungal agents, sepsis, environmental toxins, drugs of abuse, contaminants, and sepsis., ${ }^{1,2}$ The pathophysiology of TLE is not fully understood, but one mechanism may involve damage to axonal mitochondria. ${ }^{3}$ Although the prevalence of TLE is unknown, only 160 cases were reported by 2018 , and the incidence may be rising with the increasing prevalence of drug abuse..$^{4,5}$

The most common presenting symptoms of TLE are confusion, delirium, ataxia, and apraxia. ${ }^{6}$ However, the clinical presentation of TLE is variable and broad. ${ }^{4}$ For example, near complete lack of responsiveness has been reported in a case of TLE in a female with a history of psychosis and catatonia. ${ }^{7} \mathrm{~A}$ better understanding of the breadth of clinical presentation and neurological imaging may help with earlier identification. This case shows that TLE should be included in the differential diagnosis for patients with a history of substance abuse and neurobehavioral changes, including agitation and social withdrawal.

TLE rarely occurs, and it is important to consider a broad differential diagnosis when caring for patients with new-onset neuropsychiatric clinical findings in the hospital. The differential diagnosis includes metabolic derangements, subclinical seizures, demyelinating processes, or autoimmune disorders. Evaluation should involve basic laboratory tests; blood cultures; testing for TSH levels, vitamin B12 levels, treponemal antibodies, and arterial blood gas; and neuroimaging, including brain MRI, lumbar puncture, and electroencephalogram (EEG).

The diagnosis of TLE is based on a combination of acute to subacute neuropsychiatric clinical findings in the setting of exogenous exposures and is supported by characteristic neuroimaging. In this patient, the diffuse changes in subcortical white matter seen on T2/FLAIR brain MRI suggest TLE, particularly in the setting of substance abuse. However, imaging tests may reveal other insults, such as genetic metabolic diseases and subacute hypoxic-ischemic encephalopathy. ${ }^{9,10}$

The prognosis for TLE depends on the severity of symptoms. In a review of 83 patients, all patients with mild cases of TLE (characterized by confusion, attention, and ataxia) survived and may retain independence for activities of daily living; ${ }^{6} 41$ out of 42 patients with moderate cases of TLE (characterized by corticospinal or extrapyramidal involvement with severe confusion or delirium) survived; and only 8 out of $24(33 \%)$ patients with severe cases of TLE (characterized by generalized motor impairment and abulia) survived. ${ }^{6}$

Treatment for TLE is limited to supportive care and approaches described in case reports and small observational studies. Several cases suggest that coenzyme Q, ${ }^{11}$ tetrahydrobiopterin $(\mathrm{BH} 4),{ }^{3,6}$ and antioxidants $^{12}$ can aid in the recovery of some TLE patients. These treatments may work by reversing oxidative damage to axonal mitochondria. ${ }^{3,12}$ Other studies suggest using immunotherapy, including prednisone and immunoglobulin, ${ }^{13,14}$ which may suppress or modulate the immune system and reduce inflammation and brain edema. ${ }^{13}$ More research is needed to further characterize the therapeutic role of antioxidant and anti-inflammatory modalities in TLE.

Due to limited treatment options for this rare but increasingly prevalent disease, it is important to remove offending agents. Because substance abuse is a major cause of TLE, increased efforts are needed to identify and treat patients with substance use disorder. In patients with a history of substance abuse and delirium, including social withdrawal and agitation, TLE should be included in the differential diagnosis and a brain MRI should not be delayed. Early diagnosis may help to remove offending agents, whether iatrogenic or recreational drugs. ${ }^{8}$ 
Figure 1. Axial T2-weighted $(A)$ and fluid-attenuated inversion recovery $(B)$ of the brain showing diffuse changes in subcortical white matter (arrows).
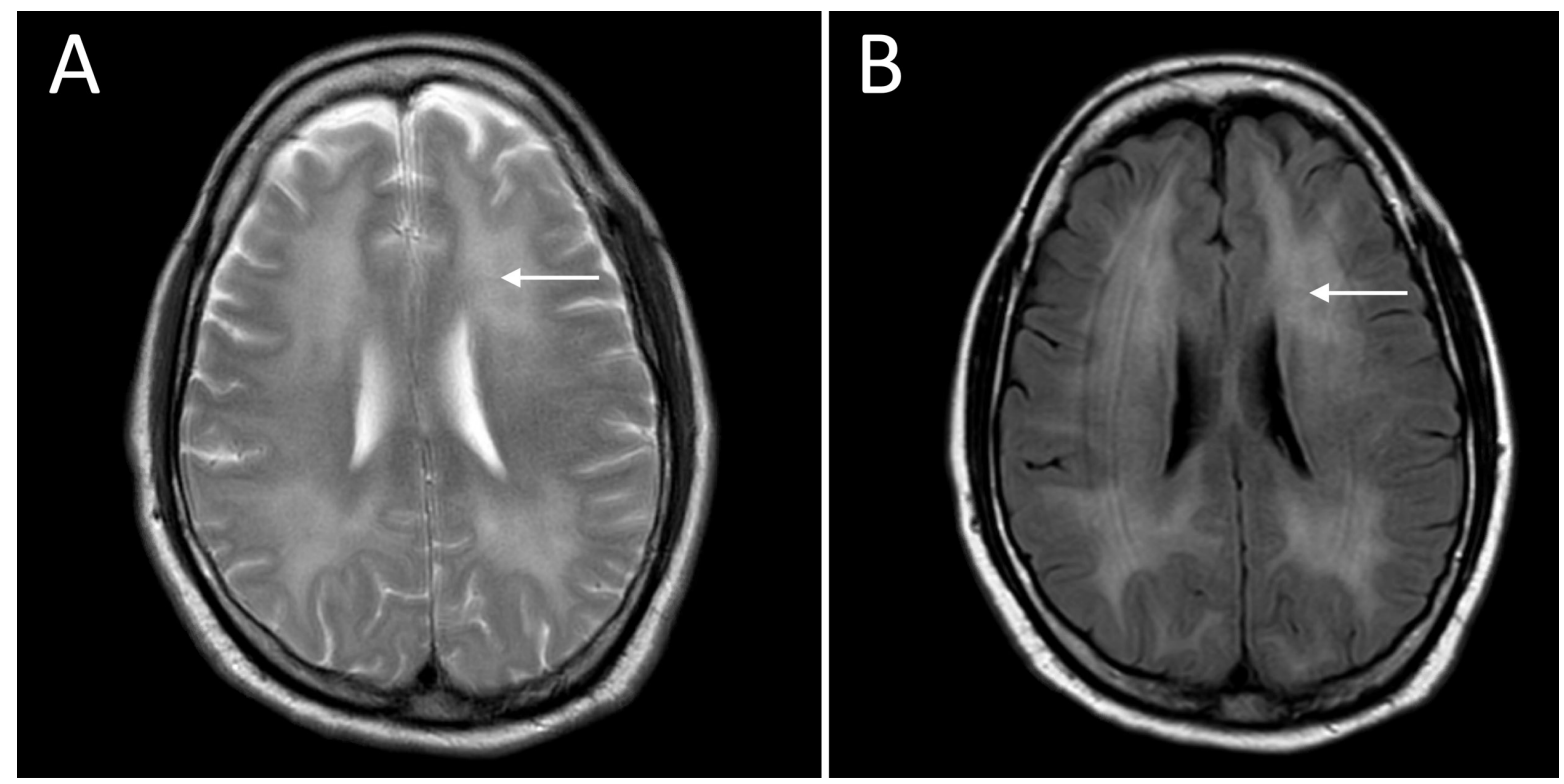

\section{Conflicts of Interest: None}

\section{REFERENCES}

1. Filley CM. Toxic leukoencephalopathy. Clin Neuropharmacol. 1999;22(5):249-260.

2. Crossen JR, Garwood D, Glatstein E, Neuwelt EA. Neurobehavioral sequelae of cranial irradiation in adults: a review of radiation-induced encephalopathy. J Clin Oncol. 1994;12(3):627-642. doi:10.1200/JCO.1994.12.3.627

3. Heales S, Crawley F, Rudge P. Reversible parkinsonism following heroin pyrolysate inhalation is associated with tetrahydrobiopterin deficiency. Move Disord. 2004;19(10):1248-1251. doi:10.1002/ mds. 20158

4. Filley CM, McConnell BV, Anderson CA. The expanding prominence of toxic leukoencephalopathy. $J$ Neuropsychiatry Clin Neurosci. 2017;29(4):308-318. doi:10.1176/appi. neuropsych.17010006

5. U.S. Department of Health and Human Services, Substance Abuse and Mental Health Services Administration, Center for Behavioral Health Statistics and Quality. Key substance use and mental health indicators in the United States: results from the 2015 National Survey on Drug Use and Health. Retrieved from https:// datafiles.samhsa.gov/. Accessed September 27, 2019.

6. Alambyan V, Pace J, Miller B, et al. The emerging role of inhaled heroin in the opioid epidemic: a review. JAMA Neurol. 2018;75(11):1423-1434. doi:10.1001/jamaneurol.2018.1693

7. van Esch AMJ, Fest A, Hoffland BS, et al. Toxic leukoencephalopathy presenting as lethal catatonia. J Addict Med. 2019;13(3):241-244. doi:10.1097/ADM.0000000000000470

8. Maheen Anwar SS, Mubarak F, Sajjad Z, Azeemuddin M. 5-FU induced acute toxic leukoencephalopathy: early recognition and reversibility on DWI-MRI. J Coll Physicians Surg Pak. 2014;24 Suppl 1:S8-10. doi:03.2014/JCPSP.S8S10

9. Patay Z. Diffusion-weighted MR imaging in leukodystrophies. Eur Radiol. 2005;15(11):2284-2303. doi:10.1007/s00330-0052846-2

10. Finelli PF, DiMario FJ Jr. MR imaging and prognosis of hypoxicischemic leukoencephalopathy. Neurocrit Care. 2006;4(2):119126. doi:10.1385/NCC:4:2:119

11. Gacouin A, Lavoue S, Signouret T, et al. Reversible spongiform leucoencephalopathy after inhalation of heated heroin. Intensive Care Med. 2003;29(6):1012-1015. doi:10.1007/s00134-0031691-5

12. KriegsteinAR, Shungu DC, MillarWS, et al. Leukoencephalopathy and raised brain lactate from heroin vapor inhalation ("chasing the dragon"). Neurology. 1999;53(8):1765-1773. doi:10.1212/ wnl.53.8.1765

13. Mader EC Jr, Ramos AB, Cruz RA, Branch LA. Full recovery from cocaine-induced toxic leukoencephalopathy: emphasizing the role of neuroinflammation and brain edema. J Investig Med High Impact Case Rep. 2019;7: 2324709619868266. doi:10.1177/2324709619868266

14. Vosoughi R, Schmidt BJ. Multifocal leukoencephalopathy in cocaine users: a report of two cases and review of the literature. BMC Neurol. 2015;15:208. doi:10.1186/s12883-015-0467-1 